\title{
WEIGHTED NORM INEQUALITIES FOR THE RIEMANN-LIOUVILLE AND WEYL FRACTIONAL INTEGRAL OPERATORS
}

\author{
K. F. ANDERSEN AND E. T. SAWYER
}

\begin{abstract}
The weight functions $u(x)$ for which $R_{\alpha}$, the Riemann-Liouville fractional integral operator of order $\alpha>0$, is bounded from $L^{p}\left(u^{p} d x\right)$ to $L^{q}\left(u^{q} d x\right), 1<p<1 / \alpha, 1 / q=1 / p-\alpha$, are characterized. Further, given $p, q$ with $1 / q \geq 1 / p-\alpha$, the weight functions $u>0$ a.e. (resp. $v<\infty$ a.e.) for which there is $v<\infty$ a.e. (resp. $u>0$ a.e.) so that $R_{\alpha}$ is bounded from $L^{p}\left(v^{p} d x\right)$ to $L^{q}\left(u^{q} d x\right)$ are characterized. Analogous results are obtained for the Weyl fractional integral. The method involves the use of complex interpolation of analytic families of operators to obtain similar results for fractional "one-sided" maximal function operators which are of independent interest.
\end{abstract}

1. Introduction. For $0<\alpha<1$ the Riemann-Liouville fractional integral operator $R_{\alpha}$ and the Weyl fractional integral operator $W_{\alpha}$ are defined, up to normalizing constants, for locally integrable functions $f$ on $(0, \infty)$ by

$$
\left(R_{\alpha} f\right)(x)=\int_{0}^{x}(x-t)^{\alpha-1} f(t) d t, \quad\left(W_{\alpha} f\right)(x)=\int_{x}^{\infty}(t-x)^{\alpha-1} f(t) d t, \quad x>0 .
$$

If $1<p<1 / \alpha, 1 / q=1 / p-\alpha$ and $T$ is either one of these operators, then it is well known [7, Theorem 383] that

$$
\left[\int_{0}^{\infty}|(T f)(x)|^{q} d x\right]^{1 / q} \leq C\left[\int_{0}^{\infty}|f(x)|^{p} d x\right]^{1 / p}
$$

for some constant $C$ depending on $p, q, \alpha$ but independent of $f$. The purpose of this paper is to study weighted analogues of these inequalities of the form

$$
\left[\int_{0}^{\infty}|(T f)(x) u(x)|^{q} d x\right]^{1 / q} \leq C\left[\int_{0}^{\infty}|f(x) v(x)|^{p} d x\right]^{1 / p}
$$

where $u$ and $v$ are nonnegative weight functions and $C$ is a constant depending on $p, q, \alpha, u, v$ but independent of $f$.

Our approach to these inequalities involves dominating $R_{\alpha}$ and $W_{\alpha}$ in terms of the fractional "one-sided" maximal function operators $M_{\alpha}^{-}$and $M_{\alpha}^{+}$, defined for

Received by the editors December 5, 1986.

1980 Mathematics Subject (lassification (1985 Revision). Primary 26A33; Secondary 26D10; 42B25.

Research of the first author supported in part by NSERC grant A8185.

Research of the second author supported in part by NSERC grant A5149. 
$0 \leq \alpha<1$ by

$$
\begin{aligned}
& \left(M_{\alpha}^{-} f\right)(x)=\sup _{0<h<x} h^{\alpha-1} \int_{x-h}^{x}|f(t)| d t, \quad x>0 \\
& \left(M_{\alpha}^{+} f\right)(x)=\sup _{h>0} h^{\alpha-1} \int_{x}^{x+h}|f(t)| d t, \quad x>0 .
\end{aligned}
$$

As is easy to see, $\left(M_{\alpha}^{-} f\right)(x) \leq\left(R_{\alpha}|f|\right)(x)$ and $\left(M_{\alpha}^{+} f\right)(x) \leq\left(W_{\alpha}|f|\right)(x)$. Even though these inequalities cannot be reversed, applying a result of Welland [15] to the function $f(t) \chi_{(0, x)}(t)$, it follows that for each $\varepsilon, 0<\varepsilon<\min (\alpha, 1-\alpha)$, there is a constant $C_{\varepsilon}$ depending only on $\varepsilon$, such that

$$
\left|\left(R_{\alpha} f\right)(x)\right| \leq C_{\varepsilon}\left[\left(M_{\alpha+\varepsilon}^{-} f\right)(x)\left(M_{\alpha-\varepsilon}^{-} f\right)(x)\right]^{1 / 2} .
$$

Similarly

$$
\left|\left(W_{\alpha} f\right)(x)\right| \leq C_{\varepsilon}\left[\left(M_{\alpha+\varepsilon}^{+} f\right)(x)\left(M_{\alpha-\varepsilon}^{+} f\right)(x)\right]^{1 / 2} .
$$

From (1.2), (1.3) and the results we prove for (1.1) with $T=M_{\alpha}^{-}$and $T=M_{\alpha}^{+}$, which are of interest in their own right, we shall deduce inequalities of the form (1.1) for $T=R_{\alpha}$ and $T=W_{\alpha}$. From these, the interested reader may easily deduce (see [2]) inequalities of the form (1.1) for various other fractional integral operators $T$ such as the Erdélyi-Kober operators $I_{\alpha, \xi}^{\nu}$ and $J_{\beta, \eta}^{\nu}$ given by

$$
\begin{aligned}
& \left(I_{\alpha, \xi}^{\nu} f\right)(x)=\frac{\nu x^{-\nu(\xi+\alpha)+\nu}}{\Gamma(\alpha)} \int_{0}^{x}\left(x^{\nu}-t^{\nu}\right)^{\alpha-1} t^{\nu \xi-1} f(t) d t \\
& \left(J_{\beta, \eta}^{\nu} f\right)(x)=\frac{\nu x^{\nu \eta}}{\Gamma(\alpha)} \int_{x}^{\infty}\left(t^{\nu}-x^{\nu}\right)^{\beta-1} t^{-\nu(\beta+\eta)+\nu-1} f(t) d t
\end{aligned}
$$

Under the hypothesis $1<p<1 / \alpha, 1 / q=1 / p-\alpha$ and $u=v$, we give characterizations of those weights $u$ for which (1.1) holds if $T$ is any one of $R_{\alpha}, W_{\alpha}, M_{\alpha}^{-}$, $M_{\alpha}^{+}$. Indeed, we shall prove the following theorem.

THEOREM 1. If $0 \leq \alpha<1,1<p<1 / \alpha(1 / \alpha=\infty$ if $\alpha=0), 1 / q=1 / p-\alpha$, $1 / p+1 / p^{\prime}=1$ then (1.1) holds with $u=v$

(a) for $T=M_{\alpha}^{-}$or $T=R_{\alpha}(\alpha>0)$ if and only if

$$
\left[\frac{1}{h} \int_{a}^{a+h} u(x)^{q} d x\right]^{1 / q}\left[\frac{1}{h} \int_{a-h}^{a} u(x)^{-p^{\prime}} d x\right]^{1 / p^{\prime}} \leq C
$$

for some constant $C$ and all $a, h$ with $0<h<a$;

(b) for $T=M_{\alpha}^{+}$or $T=W_{\alpha}(\alpha>0)$ if and only if

$$
\left[\frac{1}{h} \int_{a-h}^{a} u(x)^{q} d x\right]^{1 / q}\left[\frac{1}{h} \int_{a}^{a+h} u(x)^{-p^{\prime}} d x\right]^{1 / p^{\prime}} \leq C
$$

for some constant $C$ and all $a, h$ with $0<h<a$.

Observe in particular that any nonincreasing weight function $u$ satisfies (1.4) while any nondecreasing weight function $u$ satisfies (1.5).

The proofs of (a) and (b) are similar. For $T=M_{\alpha}^{-}$the proof applies the method, due to Stein [14], of complex interpolation of analytic families of operators to certain 
linearizations of $M_{\alpha}^{-}$. The endpoint $\alpha=1$ is trivially estimated, while the endpoint $\alpha=0$ is treated by known results [11] for $M_{0}^{-}$. The use of interpolation here is in contrast to the 'geometric' Calderón-Zygmund decomposition type methods by which the fractional Hardy-Littlewood maximal function operator $M_{\alpha}$ and the Riesz fractional integral $I_{\alpha}$ have been successfully studied, even in the more general two weight function setting $[\mathbf{1 3}, \mathbf{1 0}]$. It is desirable to obtain a 'geometric' type proof of Theorem 1, since the interpolation method seems restricted to the single weight function context, but we have so far been unable to do so. While we have not been able to characterize the weight pairs $(u, v)$ for which (1.1) holds with $T=R_{\alpha}$, $W_{\alpha}, M_{\alpha}^{-}$or $M_{\alpha}^{+}$, the following results provide answers to a pair of related problems posed by Muckenhoupt [8], namely: given $T$, if $u(x)>0$ a.e. (respectively $v(x)<\infty$ a.e.) when is there a weight function $0 \leq v(x)<\infty$ a.e. (respectively $u(x)>0$ a.e.) such that (1.1) holds? We shall prove the following two theorems.

THEOREM 2. Let $0 \leq \alpha<1,1<p, q<\infty, 1 / q \geq 1 / p-\alpha, 1 / p+1 / p^{\prime}=1$. If $u(x) \geq 0$ a.e., there is $0 \leq v(x)<\infty$ a.e. such that $(1.1)$ holds

(a) for $T=M_{\alpha}^{-}$or $T=R_{\alpha}(\alpha>0)$ if and only if

$$
\int_{R}^{\infty} \frac{u(x)^{q}}{x^{(1-\alpha) q}} d x<\infty \quad \text { for all } R>0
$$

(b) for $T=M_{\alpha}^{+}$or $T=W_{\alpha}(\alpha>0)$ if and only if

$$
\int_{0}^{R} u(x)^{q} d x<\infty \text { for all } R>0 .
$$

THEOREM 3. Let $0 \leq \alpha<1,1<p, q<\infty, 1 / q \geq 1 / p-\alpha, 1 / p+1 / p^{\prime}=1$. If $0 \leq v(x)<\infty$ a.e., there is $u(x)>0$ a.e. such that (1.1) holds

(a) for $T=M_{\alpha}^{-}$or $T=R_{\alpha}(\alpha>0)$ if and only if

$$
\int_{0}^{R} v(x)^{-p^{\prime}} d x<\infty \text { for all } R>0 .
$$

(b) for $T=W_{\alpha}(\alpha>0)$ if and only if

$$
\int_{R}^{\infty} x^{(\alpha-1) p^{\prime}} v(x)^{-p^{\prime}} d x<\infty \quad \text { for all } R>0 ;
$$

(c) for $T=M_{\alpha}^{+}$if and only if

$$
\sup _{S>R} S^{(\alpha-1) p^{\prime}} \int_{R}^{S} v(x)^{-p^{\prime}} d x<\infty \quad \text { for all } R>0 .
$$

The assertions of Theorem 3 regarding $R_{\alpha}$ and $W_{\alpha}$ follow immediately from Theorem 2 together with Hölder's inequality and its converse since $R_{\alpha}$ and $W_{\alpha}$ are dual operators in the sense that

$$
\int_{0}^{\infty}\left(R_{\alpha} f\right)(x) g(x) d x=\int_{0}^{\infty} f(x)\left(W_{\alpha} g\right)(x) d x
$$

for all $f, g \geq 0$, while $1 / q \geq 1 / p-\alpha$ if and only if $1 / p^{\prime} \geq 1 / q^{\prime}-\alpha$.

For the Hardy-Littlewood maximal operator $M$ and $p=q$, an analogue of Theorem 2 was obtained by A. Gatto and C. Gutiérrez [6] and independently by W.-S. Young [16] while an analogue of Theorem 3 was obtained by Carleson and Jones 
[5] in this case. Extensions to certain fractional maximal functions and integrals were given in [1 and 12].

Theorems 2 and 3 are proved by considering separately the local and global behaviour of $R_{\alpha}, W_{\alpha}, M_{\alpha}^{-}$and $M_{\alpha}^{+}$: the local behaviour being governed by the operator

$$
\left(N_{\alpha} f\right)(x)=\sup _{0<h<x / 4}(2 h)^{\alpha-1} \int_{x-h}^{x+h}|f(t)| d t, \quad x>0,
$$

while the global behaviour is captured by the Hardy operators

$$
(P f)(x)=\int_{0}^{x} f(t) d t \quad \text { and } \quad(Q f)(x)=\int_{x}^{\infty} f(t) d t, \quad x>0 .
$$

For example, the left fractional maximal function $M_{\alpha}^{-}$satisfies

$$
\left(M_{\alpha}^{-} f\right)(x) \leq 2^{1-\alpha}\left(N_{\alpha} f\right)(x)+4^{1-\alpha} x^{\alpha-1}(P|f|)(x) .
$$

As we shall see, conditions (1.6)-(1.10), answering the two problems posed by Muckenhoupt, are determined solely by the global behaviour. The local operator $N_{\alpha}$ is handled by the following variant of an inequality of Gatto and Gutiérrez [6]. Let

$$
(L f)(x)=\sup _{\substack{a, b>0 \\ b+a<2 x / 3}}(b+a)^{-1} \int_{x-a}^{x+b}|f(t)| d t .
$$

LEMMA 1. Let $0 \leq \alpha<1,1 \leq p<1 / \alpha(1 / \alpha=\infty$ if $\alpha=0), 1 / q=1 / p-\alpha$. There are constants $C_{p, \alpha}$ such that

$$
\int_{\left\{x>0:\left(N_{\alpha} f\right)(x)>\lambda\right\}} g(x) d x \leq C_{p, \alpha} \lambda^{-q}\left[\int_{0}^{\infty}|f(x)|^{p}[(L g)(x)]^{1-\alpha p} d x\right]^{q / p}
$$

and if $p>1$,

$$
\left[\int_{0}^{\infty}\left|\left(N_{\alpha} f\right)(x)\right|^{q} g(x) d x\right]^{1 / q} \leq C_{p, \alpha}\left[\int_{0}^{\infty}|f(x)|^{p}[(L g)(x)]^{1-\alpha p} d x\right]^{1 / p}
$$

holds for all locally integrable $f, g$ with $g>0$ a.e.

Using Lemma 1 we answer the two problems posed by Muckenhoupt in the case $T=N_{\alpha}$.

LEMMA 2. Let $0 \leq \alpha<1,1<p<1 / \alpha(1 / \alpha=\infty$ if $\alpha=0), 1 / q=1 / p-\alpha$, $1 / p+1 / p^{\prime}=1$. If $u(x)>0$ (respectively $v(x)<\infty$ ) a.e., there is $v(x)<\infty$ (respectively $u(x)>0$ ) a.e. such that (1.1) holds with $T=N_{\alpha}$, if and only if $u(x)^{q}$ $\left(\right.$ respectively $\left.v(x)^{-p^{\prime}}\right)$ is locally integrable on $(0, \infty)$, i.e. integrable on every compact subset of $(0, \infty)$.

Since the local integrability of $u(x)^{q}$ and $v(x)^{-p^{\prime}}$ is a (readily deduced) necessary condition for (1.1) to hold with $T=R_{\alpha}, W_{\alpha}, M_{\alpha}^{-}$or $M_{\alpha}^{+}$, Lemma 2 shows that the local behaviour of these operators does not play a crucial role in Theorems 2 and 3. Instead, it is the analogue of Lemma 2 for $T=P$ and $T=Q$ that is decisive. However, for $T=P$ or $T=Q$, the weights $u, v$ for which (1.1) holds have been 
characterized very simply by Bradley [4], see also [3]. For example, (1.1) holds with $T=P$ if and only if

$$
\left[\int_{R}^{\infty} u(x)^{q} d x\right]^{1 / q}\left[\int_{0}^{R} v(x)^{-p^{\prime}} d x\right]^{1 / p^{\prime}} \leq C \text { for all } R>0,
$$

and the analogue of Lemma 2 for $T=P$ is now evident (for example, given $u(x)>0$ a.e., there is $v(x)<\infty$ a.e. such that (1.1) holds with $T=P$, if and only if the first factor on the left side of (1.13) is finite for all $R>0$ ).

Theorem 1 is proved in $\S \S 2$ and 3 . The lemmas are proved in $\S \S 4$ and 5 while the remaining theorems are proved in $\S 6$. We follow the usual practice that $C$ denotes an absolute constant, not necessarily the same from line to line.

2. Proof of Theorem 1 for $T=M_{\alpha}^{-}$and $M_{\alpha}^{+}$. First suppose (1.1) holds with $T=M_{\alpha}^{-}$and $u=v$, and fix $0<h<a$. Let $f(x)=\chi_{(a-h, a)}(x) u(x)^{-p^{\prime}}$. Then for $a \leq x \leq a+h$,

$$
\left(M_{\alpha}^{-} f\right)(x) \geq(2 h)^{\alpha-1} \int_{a-h}^{a} u^{-p^{\prime}}
$$

and plugging this estimate into (1.1) we obtain

$$
(2 h)^{\alpha-1}\left[\int_{a-h}^{a} u^{-p^{\prime}}\right]\left[\int_{a}^{a+h} u^{q}\right]^{1 / q} \leq C\left[\int_{a-h}^{a} u^{-p^{\prime}}\right]^{1 / p}
$$

since $u^{-p^{\prime} p+p}=u^{-p^{\prime}}$. A standard argument [9] shows that the right side of $(2.1)$ is finite, and if we then divide both sides by this quantity, we obtain (1.4) since $1 / q+1 / p^{\prime}=1-\alpha$.

Conversely, suppose (1.4) holds and set $w=u^{1 /(1-\alpha)}$. If $0<\varphi(x)<x$ for $x>0$, $z \in \mathbf{C}$, define an operator $S_{\varphi, w}^{z}$ by

$$
\left(S_{\varphi, w}^{z} f\right)(x)=\left(\frac{w(x)}{\varphi(x)}\right)^{1-z} \int_{x-\varphi(x)}^{x} w^{z-1} f
$$

for appropriate $f$. The family of operators $\left\{S_{\varphi, w}^{z}\right\}_{z \in \mathbf{C}}$ is analytic in the open strip $\{z: 0<\operatorname{Re} z<1\}$ and continuous on the closed strip $\{z: 0 \leq \operatorname{Re} z \leq 1\}$ in the sense that each of the functions $z \mapsto \int_{-\infty}^{\infty}\left(S_{\varphi, w}^{z} f\right) g$ is analytic in the open strip and continuous on the closed strip for all $f, g$ bounded with compact support contained in the support of $w$. Note that by (1.4), the support of $w$ is of the form $[0, b], b \leq \infty$, and that $w^{-1}$ (even $w^{-(1-\alpha) p^{\prime}}$ ) is locally integrable on $(0, b)$. This is sufficient for the continuity of the family of operators (2.2) near $\operatorname{Re} z=0$.

Now set $r=q(1-\alpha)$. Then (1.4) can be rewritten

$$
\left[\frac{1}{h} \int_{a}^{a+h} w^{r}\right]^{1 / r}\left[\frac{1}{h} \int_{a-h}^{a} w^{-r^{\prime}}\right]^{1 / r^{\prime}} \leq C_{1}^{1 / 1-\alpha}
$$

since $1 / p^{\prime}=1-1 / p=1-(1 / q+\alpha)=1-(((1-\alpha) / r)-\alpha)=(1-\alpha)\left(1 / r^{\prime}\right)$. However, (2.3) and Theorem 1 of [11] imply that

$$
\int_{0}^{\infty}\left|w\left(M_{0}^{-} f\right)\right|^{r} \leq C_{2} \int_{0}^{\infty}|w f|^{r} \text { for all } f \geq 0
$$


where $C_{2}$ depends only on $C_{1}$. With $g=w f$, this becomes

$$
\int_{0}^{\infty}\left|w\left(M_{0}^{-} w^{-1} g\right)\right|^{r} \leq C_{2} \int_{0}^{\infty}|g|^{r} \quad \text { for all } g \geq 0 .
$$

Finally, since

$$
\left|\left(S_{\varphi, w}^{i y} f\right)(x)\right| \leq \frac{w(x)}{\varphi(x)} \int_{x-\varphi(x)}^{x} w^{-1}|f| \leq w\left(M_{0}^{-} w^{-1} f\right)(x) \text { for all } x,
$$

(2.4) shows that

$$
S_{\varphi, w}^{i y}: L^{r} \rightarrow L^{r} \quad \text { for all } y \in \mathbf{R}
$$

with a bound depending on $C_{1}$ but not on $y, \varphi$ or $w$.

Since $\left|\left(S_{\varphi, w}^{1+i y} f\right)(x)\right| \leq \int_{x-\varphi(x)}^{x}|f| \leq\|f\|_{L^{1}}$, we have

$$
S_{\varphi, w}^{1+i y}: L^{1} \rightarrow L^{\infty} \quad \text { for all } y \in \mathbf{R}
$$

with bound one. By complex interpolation of an analytic family of operators (Stein $[14]),(2.5)$ and $(2.6)$ yield

$$
S_{\varphi, w}^{\alpha}: L^{p} \rightarrow L^{q}
$$

with a bound depending on $C_{1}$ but not on $\varphi$ or $w$ since $1 / p=(1-\alpha)(1 / r)+\alpha$ and $1 / q=(1-\alpha)(1 / r)$.

Now fix $f(x) \geq 0$ on $(0, \infty)$ and define $0<\varphi(x)<x$ so that $\left(M_{\alpha}^{-} f\right)(x) \leq$ $2 \varphi(x)^{\alpha-1} \int_{x-\varphi(x)}^{x} f$ for almost all $x$. (We may assume $M_{\alpha}^{-} f<\infty$ a.e.). Then with $g=w^{1-\alpha} f$, we have

$$
w^{1-\alpha}\left(M_{\alpha}^{-} w^{\alpha-1} g\right)(x) \leq 2\left(S_{\varphi, w}^{\alpha} g\right)(x) \quad \text { for a.e. } x .
$$

Combining (2.7) and (2.8) we obtain

$$
\left[\int_{0}^{\infty}\left|w^{1-\alpha}\left(M_{\alpha}^{-} w^{\alpha-1} g\right)\right|^{q}\right]^{1 / q} \leq C_{3}\left[\int_{0}^{\infty}|g|^{p}\right]^{1 / p} \quad \text { for all } g \geq 0
$$

or

$$
\left[\int_{0}^{\infty}\left|u\left(M_{\alpha}^{-} f\right)\right|^{q}\right]^{1 / q} \leq C_{3}\left[\int_{0}^{\infty}|u f|^{p}\right]^{1 / p} \quad \text { for all } f \geq 0
$$

where $C_{3}$ depends on $C_{1}$ but not on $f$. This proves (1.1) for $T=M_{\alpha}^{-}$and $u=v$ and completes the proof of part (a) of Theorem 1 in the case $T=M_{\alpha}^{-}$. The proof of part (b) in the case $T=M_{\alpha}^{+}$is similar.

3. Proof of Theorem 1 for $T=R_{\alpha}$ and $W_{\alpha}$. We prove only the assertion regarding $R_{\alpha}$ in part (a), the proof for $W_{\alpha}$ being similar. First, since $\left(M_{\alpha}^{-} f\right)(x) \leq$ $\left(R_{\alpha}|f|\right)(x)$, the necessity of (1.4) for (1.1) with $T=R_{\alpha}$ and $u=v$ follows immediately from what was proved in $\S 2$. Conversely, assume (1.4) holds. We will deduce (1.1) for $T=R_{\alpha}$ and $u=v$ from Welland's inequality, (1.2), and the results just proved for $M_{\alpha}^{-}$in $\S 2$. To this end, we set $\alpha_{1}=\alpha-\varepsilon, \alpha_{2}=\alpha+\varepsilon$. For $i=1,2$, 
define $q_{i}$ by $1 / q_{i}=1 / p-\alpha_{i}$, so that $1 / 2 q_{1}+1 / 2 q_{2}=1 / q$. Now apply $(1.2)$ and Hölder's inequality with indices $2 q_{1} / q$ and $2 q_{2} / q$ to obtain

$$
\begin{aligned}
& {\left[\int_{0}^{\infty}\left|\left(R_{\alpha} f\right)(x)\right|^{q} u(x)^{q} d x\right]^{1 / q}} \\
& \quad \leq C_{\varepsilon}\left[\int_{0}^{\infty}\left|\left(M_{\alpha_{1}}^{-} f\right)(x)\right|^{q_{1}} u(x)^{q_{1}} d x\right]^{1 / 2 q_{1}}\left[\int_{0}^{\infty}\left|\left(M_{\alpha_{2}}^{-} f\right)(x)\right|^{q_{2}} u(x)^{q_{2}} d x\right]^{1 / 2 q_{2}} \\
& \quad=C_{\varepsilon} \text { I II. }
\end{aligned}
$$

Now $q_{1}<q$ and so Hölder's inequality shows that (1.4), which we have assumed, holds also with $q_{1}$ in place of $q$. Thus $\mathrm{I} \leq C\left[\int_{0}^{\infty}|f(x)|^{p} u(x)^{p} d x\right]^{1 / 2 p}$ by the results of $\S 2$. Term II is handled in the same way once we are able to show that (1.4) holds with $q_{2}$ in place of $q$ for $\varepsilon$ sufficiently small. Note however that Hölder's inequality fails for this purpose since $q_{2}>q$. Instead, we use the theory of $A_{p}^{-}$weights in [11]. First, we observe that (1.4) is equivalent to the assertion that $u^{q}$ satisfies the $A_{r}^{-}$ condition with $r=1+q / p^{\prime}$. Thus $u^{q\left(1-r^{\prime}\right)}$ satisfies $A_{r^{\prime}}^{+}[11$, Remark A] and so also $A_{s^{\prime}}^{+}$for some $s>r\left[\mathbf{1 1}\right.$, Remark C]. By [11, Remark A] once more, $u^{q\left(1-r^{\prime}\right)(1-s)}$ satisfies $A_{s}^{-}$. Now choose $\varepsilon>0$ so that

$$
q_{2}=q\left(1-r^{\prime}\right)(1-s)=q\left(\frac{s-1}{r-1}\right) .
$$

Then $u^{q_{2}}$ satisfies $A_{s}^{-}$which is equivalent to (1.4) with $q_{2}$ in place of $q$ (since $\left.q_{2}\left(1-s^{\prime}\right)=q\left(1-r^{\prime}\right)=q /(1-r)=-p^{\prime}\right)$. As before, the results of $\S 2$ yield II $\leq C\left[\int_{0}^{\infty}|f(x)|^{p} u(x)^{p} d x\right]^{1 / 2 p}$, and combining these estimates for I and II with (3.1) yields (1.1) with $T=R_{\alpha}$ and $u=v$.

4. Proof of Lemma 1. Fix $\lambda>0$ and $f, g$ locally integrable with $g>0$. Set $E_{\lambda}=\left\{x>0:\left(N_{\alpha} f\right)(x)>\lambda\right\}$. Then $E_{\lambda} \subset \bigcup_{x \in E_{\lambda}} I_{x}$ where $I_{x}$ is an interval $\left(x-h_{x}, x+h_{x}\right)$ with $h_{x}<x / 4$ and $\left(2 h_{x}\right)^{\alpha-1} \int_{I_{x}}|f|>\lambda$. There are sequences $\left\{x_{j}\right\}$, $\left\{h_{j}\right\}\left(h_{j}=h_{x_{j}}\right)$ and a constant $C$ so that

$$
\chi_{E_{\lambda}}(t) \leq \sum_{j} \chi_{\left(x_{j}-h_{j}, x_{j}+h_{j}\right)}(t) \leq C
$$

Then,

$$
\begin{aligned}
\int_{E_{\lambda}} g(x) d x & \leq \sum_{j} \int_{x_{j}-h_{j}}^{x_{j}+h_{j}} g(x) d x \\
& \leq \sum_{j}\left[\int_{x_{j}-h_{j}}^{x_{j}+h_{j}} g(x) d x\right]\left[\frac{1}{\lambda\left(2 h_{j}\right)^{1-\alpha}} \int_{x_{j}-h_{j}}^{x_{j}+h_{j}}|f(t)| d t\right]^{q} \\
& \leq \lambda^{-q} \sum_{j}\left[\frac{1}{2 h_{j}} \int_{x_{j}-h_{j}}^{x_{j}+h_{j}} g(x) d x\right]\left[\int_{x_{j}-h_{j}}^{x_{j}+h_{j}}|f(t)|^{p} d t\right]^{q / p}
\end{aligned}
$$

by Hölder's inequality and the fact that $\left[(1-\alpha)-1 / p^{\prime}\right] q=1$. Now observe that if $t \in\left(x_{j}-h_{j}, x_{j}+h_{j}\right)$ then $t>x_{j}-h_{j}>3 h_{j}$ since $h_{j}<x_{j} / 4$, and so

$$
\frac{1}{2 h_{j}} \int_{x_{j}-h_{j}}^{x_{j}+h_{j}} g(x) d x \leq(L g)(t) .
$$


Thus,

$$
\begin{gathered}
\int_{E_{\lambda}} g(x) d x \leq \lambda^{-q} \sum_{j}\left[\int_{x_{j}-h_{j}}^{x_{j}+h_{j}}|f(t)|^{p}[(L g)(t)]^{p / q} d t\right]^{q / p} \\
\leq \lambda^{-q}\left[\sum_{j} \int_{x_{j}-h_{j}}^{x_{j}+h_{j}}|f(t)|^{p}[(L g)(t)]^{p / q} d t\right]^{q / p} \text { since } q \geq p \\
\leq C^{q / p} \lambda^{-q}\left[\int_{0}^{\infty}|f(t)|^{p}[(L g)(t)]^{p / q} d t\right]^{q / p} \text { by }(4.1),
\end{gathered}
$$

i.e.

$$
\left[\int_{E_{\lambda}} g(x) d x\right]^{1 / q} \leq C_{p, \alpha} \lambda^{-1}\left[\int_{0}^{\infty}|f(t)|^{p}[(L g)(t)]^{1-\alpha p} d t\right]^{1 / p} .
$$

Let $\left(T_{\alpha} f\right)(x)=\left(N_{\alpha}\left[f(L g)^{\alpha}\right]\right)(x)$ (for fixed $\left.g\right)$. Then (4.2) yields

$$
\left[\int_{\left\{x>0:\left(T_{\alpha} f\right)(x)>\lambda\right\}} g(x) d x\right]^{1 / q} \leq C_{p, \alpha} \lambda^{-1}\left[\int_{0}^{\infty}|f(t)|^{p}(L g)(t) d t\right]^{1 / p}
$$

and Marcinkiewicz interpolation then yields (for $p>1$ )

$$
\left[\int_{0}^{\infty}\left|N_{\alpha}\left[f(L g)^{\alpha}\right]\right|^{q} g(x) d x\right]^{1 / q} \leq C_{p, \alpha}\left[\int_{0}^{\infty}|f(t)|^{p}(L g)(t) d t\right]^{1 / p}
$$

since $L g>0$ a.e. this is equivalent to (1.12).

5. Proof of Lemma 2. We begin by proving the necessity of local integrability for $u^{q}$ and $v^{-p^{\prime}}$ if (1.1) holds. So suppose (1.1) holds with $T=N_{\alpha}$ where $u(x)>0$ a.e. and $v(x)<\infty$ a.e. For $R>0$ and $\varepsilon>0$, let $f_{R, \varepsilon}(x)=\chi_{(R, 5 R / 4)}(x)(v(x)+\varepsilon)^{-p^{\prime}}$. Then

$$
\left(N_{\alpha} f_{R, \varepsilon}\right)(x) \geq\left(\frac{R}{4}\right)^{\alpha-1} \int_{R}^{5 R / 4}(v+\varepsilon)^{-p^{\prime}}, \quad R \leq x \leq 5 R / 4
$$

and so

$$
\begin{aligned}
& \left(\frac{R}{4}\right)^{\alpha-1}\left[\int_{R}^{5 R / 4}(v+\varepsilon)^{-p^{\prime}}\right]\left[\int_{R}^{5 R / 4} u^{q}\right]^{1 / q} \leq\left[\int_{0}^{\infty}\left(N_{\alpha} f_{R, \varepsilon}\right)^{q} u^{q}\right]^{1 / q} \\
& \leq C\left[\int_{0}^{\infty}\left(f_{R, \varepsilon}\right)^{p} v^{p}\right]^{1 / p} \text { by }(1.1) \\
& \leq C\left[\int_{R}^{5 R / 4}(v+\varepsilon)^{-p p^{\prime}} v^{p}\right]^{1 / p} \\
& \leq C\left[\int_{R}^{5 R / 4}(v+\varepsilon)^{-p^{\prime}}\right]^{1 / p} \text { since } p-p p^{\prime}=-p^{\prime} .
\end{aligned}
$$

Now divide both sides of (5.1) by the term on the right side and then let $\varepsilon \rightarrow 0$ to obtain that both $u^{q}$ and $v^{-p^{\prime}}$ are integrable on $(R, 5 R / 4)$ for all $R>0$. 
Conversely, we consider first the case where $u(x)^{q}>0$ a.e. and locally integrable on $(0, \infty)$. Let $u_{n}=\chi_{\left(2^{n}, 2^{n+1}\right)} u$ for $n \in Z$. Then for $f$ locally integrable,

$$
\begin{aligned}
\int_{0}^{\infty}\left|N_{\alpha} f\right|^{q} u^{q} & \leq \sum_{n \in Z} \int_{0}^{\infty}\left|N_{\alpha} f\right|^{q}\left(u_{n}+2^{-|n|}\right)^{q} \\
& \leq C \sum_{n \in Z}\left[\int_{0}^{\infty}|f|^{p}\left(L g_{n}\right)^{1-\alpha p}\right]^{q / p} \text { by }(1.12) \text { with } g_{n}=\left(u_{n}+2^{-|n|}\right)^{q} \\
& \leq C\left[\int_{0}^{\infty}|f|^{p}\left[\sum_{n \in Z}\left(L g_{n}\right)^{1-\alpha p}\right]\right]^{q / p} \text { since } p \leq q .
\end{aligned}
$$

Thus (1.1) holds for $T=N_{\alpha}$ if we define $v(x)$ by $v^{p}=\sum_{n \in Z}\left(L g_{n}\right)^{1-\alpha p}$ and $v(x)$ is finite a.e. since the support of $L u_{n}^{q}$ is contained in $\left[2^{n-1}, 2^{n+2}\right]$ and $L u_{n}^{q}<\infty$ a.e. for each $n$.

Finally, we consider the case where $v(x)<\infty$ a.e. and $v(x)^{-p^{\prime}}$ is locally integrable on $(0, \infty)$. For notational convenience we set $\sigma=v^{-p^{\prime}}$ and for $R>0, \sigma_{R}=$ $\chi_{(R, 2 R)} \sigma$. Let

$$
E_{R, k}=\left\{x \in[4 R / 3,8 R / 5]: N_{0} \sigma_{R} \leq 2^{k}\right\}
$$

and define an operator $T_{R, k}$ by

$$
\left(T_{R, k} f\right)(x)=\chi_{E_{R, k}}(x) N_{\alpha}\left(f \sigma_{R}\right)(x) .
$$

With $g \equiv 1,(1.11)$ shows that

$$
T_{R, k}: L^{1}\left(\sigma_{R}\right) \rightarrow L^{1 /(1-\alpha), \infty}(d x) \text { with norm } \leq C,
$$

while Hölder's inequality yields

$$
T_{R, k}=L^{1 / \alpha}\left(\sigma_{R}\right) \rightarrow L^{\infty}(d x) \quad \text { with norm } \leq 2^{k(1-\alpha)}
$$

since

$$
\begin{aligned}
\left(T_{R, k} f\right)(x) & =\chi_{E_{R, k}}(x)\left[\sup _{0<h<x / 4}(2 h)^{\alpha-1} \int_{x-h}^{x+h}|f| \sigma_{R}\right] \\
& \leq \chi_{E_{R, k}}(x) \sup _{0<h<x / 4}\left[\frac{1}{2 h} \int_{x-h}^{x+h} \sigma_{R}\right]^{1-\alpha}\left[\int_{0}^{\infty}|f|^{1 / \alpha} \sigma_{R}\right]^{\alpha} \\
& \leq 2^{k(1-\alpha)}\left[\int_{0}^{\infty}|f|^{1 / \alpha} \sigma_{R}\right]^{\alpha} \text { by definition of } E_{R, k} .
\end{aligned}
$$

Applying Marcinkiewicz interpolation to (5.2) and (5.3) yields

$$
\left[\int_{0}^{\infty}\left|T_{R, k} f\right|^{q}\right]^{1 / q} \leq C_{p, \alpha} 2^{k / p^{\prime}}\left[\int_{0}^{\infty}|f|^{p} \sigma_{R}\right]^{1 / p} \quad \text { for all } f \geq 0 .
$$

Recalling that $\left(T_{R, k} f\right)(x)=\left(N_{\alpha} f v^{-p^{\prime}}\right)(x)$ for $x \in E_{R, k}$, and then replacing $f$ by $g v^{p^{\prime}}$ in (5.4) produces

$$
\left[\int_{E_{R, k}}\left|N_{\alpha} g\right|^{q}\right]^{1 / q} \leq C_{p, \alpha} 2^{k / p^{\prime}}\left[\int_{R}^{2 R}|g|^{p} v^{p}\right]^{1 / p} \quad \text { for all } g \geq 0 .
$$


Now define $u_{R}$ by

$$
u_{R}^{q}=\sum_{k=0}^{\infty} 2^{-k} 2^{-k q / p^{\prime}} \chi_{E_{R, k}} \quad\left[\approx \chi_{(4 R / 3,8 R / 5)}\left(N_{0} \sigma_{R}\right)^{-q / p^{\prime}-1}\right] .
$$

Raising (5.5) to the power $q$ and summing we obtain

$$
\int_{0}^{\infty}\left(N_{\alpha} g\right)^{q} u_{R}^{q} \leq 2 C_{p, \alpha}^{q}\left[\int_{R}^{2 R}|g|^{p} v^{p}\right]^{q / p} \quad \text { for all } g \geq 0 .
$$

Setting $R=R_{j}=\left(\frac{3}{4}\right)\left(\frac{6}{5}\right)^{j}$ in $(5.6)$, multiplying by $2^{-|j|}$ and then summing the result yields

$$
\left[\int_{0}^{\infty}\left(N_{\alpha} g\right)^{q} \sum_{j \in Z} 2^{-|j|} u_{R_{j}}^{q}\right]^{1 / q} \leq C_{p, \alpha}\left[\int_{0}^{\infty}|g|^{p} v^{p}\right]^{1 / p} \quad \text { for all } g>0 .
$$

Thus (1.1) holds for $T=N_{\alpha}$ if we define $u(x)$ by $u^{q}=\sum_{j \in Z} 2^{-|j|} u_{R_{j}}^{q}$ and $u(x)$ is positive a.e. by construction.

6. Proofs of Theorems 2 and 3. We begin by proving part (a) of Theorem 2. Suppose first that (1.1) holds for $T=R_{\alpha}$ or $T=M_{\alpha}^{-}$where $u(x)>0$ a.e. and $v(x)<\infty$ a.e. For $R>0$ and $\varepsilon>0$, let

$$
f_{R, \varepsilon}(x)=\chi_{(0, R)}(x)(v(x)+\varepsilon)^{-p^{\prime}} .
$$

Then

$$
\left(R_{\alpha} f\right)(x) \geq\left(M_{\alpha}^{-} f\right)(x) \geq x^{\alpha-1} \int_{0}^{R}(v+\varepsilon)^{-p^{\prime}}, \quad x>R,
$$

and plugging this estimate into (1.1), arguing as in (5.1) and then letting $\varepsilon \rightarrow 0$ yields

$$
\left[\int_{R}^{\infty} \frac{u(x)^{q}}{x^{(1-\alpha) q}}\right]^{1 / q}\left[\int_{0}^{R} v(x)^{-p^{\prime}} d x\right]^{1 / p^{\prime}} \leq C \text { for all } R>0 .
$$

This proves the necessity of (1.6) (and also (1.8)) for (1.1) with $T=R_{\alpha}$ or $T=M_{\alpha}^{-}$.

Conversely, suppose (1.6) holds. We assume in what follows that $1 / q=1 / p-\alpha$ since the case $1 / q>1 / p-\alpha$ may be obtained from this using Hölder's inequality in a suitable manner. As pointed out in the introduction, we have for $f \geq 0$,

$$
\left(M_{\alpha}^{-} f\right)(x) \leq 2^{1-\alpha}\left(N_{\alpha} f\right)(x)+4^{1-\alpha} x^{\alpha-1}(P f)(x), \quad x>0 .
$$

By Lemma 2, there is $v_{1}(x)<\infty$ a.e. such that (1.1) holds for $T=N_{\alpha}$ with $v=v_{1}$. If we choose $v_{2}$ to satisfy

$$
\left[\int_{R}^{\infty} x^{(\alpha-1) q} u(x)^{q} d x\right]^{1 / q}\left[\int_{0}^{R} v_{2}^{-p^{\prime}}\right]^{1 / p^{\prime}}=1 \text { for all } R>0
$$


then Bradley's result (see (1.12)) shows that (1.1) holds for $(T f)(x)=x^{\alpha-1}(P f)(x)$ with $v=v_{2}$. Thus if $v=\max \left\{v_{1}, v_{2}\right\}$, then (6.2) shows that (1.1) holds for $T=M_{\alpha}^{-}$.

To show that (1.1) holds for $T=R_{\alpha}$ (with some $v(x)<\infty$ a.e.) we use Welland's inequality, (1.2), as in the proof of Theorem 1. Choose $\varepsilon>0$ so that $\varepsilon<\min \{\alpha, 1 / p-\alpha\}$ and set $1 / q_{1}=1 / p-(\alpha-\varepsilon), 1 / q_{2}=1 / p-(\alpha+\varepsilon)$. Note that

$$
\begin{aligned}
\int_{R}^{\infty} \frac{u(x)^{q}}{x^{[1-(\alpha-\varepsilon)] q_{1}+\varepsilon q q_{1} / p^{\prime}}} d x & =\int_{R}^{\infty} \frac{u(x)^{q}}{x^{[1-(\alpha+\varepsilon)] q_{2}-\varepsilon q q_{2} / p^{\prime}}} d x \\
& =\int_{R}^{\infty} \frac{u(x)^{q}}{x^{(1-\alpha) q}} d x<\infty \text { for all } R>0 .
\end{aligned}
$$

By what we just proved for $M_{\alpha}^{-}$, there are $v_{1}(x)$ and $v_{2}(x)$ finite a.e. so that

$$
\begin{gathered}
{\left[\int_{0}^{\infty}\left[\left(M_{\alpha-\varepsilon}^{-} f\right)(x)\right]^{q_{1}} \frac{u(x)^{q}}{x^{\varepsilon q q_{1} / p^{\prime}}} d x\right]^{1 / q_{1}} \leq\left[\int_{0}^{\infty}|f|^{p} v_{1}\right]^{1 / p}} \\
{\left[\int_{0}^{\infty}\left[\left(M_{\alpha+\varepsilon}^{-} f\right)(x)\right]^{q_{2}} \frac{u(x)^{q}}{x^{-\varepsilon q q_{2} / p^{\prime}}} d x\right]^{1 / q_{2}} \leq\left[\int_{0}^{\infty}|f|^{p} v_{2}\right]^{1 / p},}
\end{gathered}
$$

for all $f \geq 0$. Applying (1.2) and Hölder's inequality with exponents $2 q_{1} / q$ and $2 q_{2} / q$, we see that $\left[\int_{0}^{\infty}\left|\left(R_{\alpha} f\right)(x)\right|^{q} u(x)^{q} d x\right]^{1 / q}$ is dominated by a constant multiple of the geometric mean of (6.3) and (6.4). Thus (1.1) holds for $T=R_{\alpha}$ with $v=\max \left\{v_{1}, v_{2}\right\}$. This proves part (a) of Theorem 2. The remaining cases of Theorems 2 and 3 are all proved similarly, i.e. $M_{\alpha}^{-}$and $M_{\alpha}^{+}$are dominated by $N_{\alpha}$ and $P$ or $Q$, and then Welland's inequalities, (1.2) and (1.3), are used to pass to $R_{\alpha}$ and $W_{\alpha}$.

\section{REFERENCES}

1. K. F. Andersen, Weighted inequalities for fractional integrals, Fractional Calculus, Res. Notes Math. 138, Pitman, 1985, pp. 12-25.

2. K. F. Andersen and H. P. Heinig, Weighted norm inequalities for certain integral operators, SIAM J. Math. Anal. 14 (1983), 833-844.

3. K. F. Andersen and B. Muckenhoupt, Weighted weak type Hardy inequalities with applications to Hilbert transforms and maximal functions, Studia Math. 72 (1982), 9-26.

4. J. S. Bradley, Hardy inequalities with mixed norms, Canad. Math. Bull. 21 (1978), 405-408.

5. L. Carleson and P. Jones, Weighted norm inequalities and a theorem of Koosis, Mittag-Leffler Inst. Rep. 2 (1981).

6. A. Gatto and C. Gutiérrez, On weighted norm inequalities for the maximal function, Studia Math. 76 (1983), 59-62.

7. G. H. Hardy, J. E. Littlewood and G. Polya, Inequalities, Cambridge Univ. Press, 1967.

8. B. Muckenhoupt, Weighted norm inequalities for classical operators, Proc. Sympos. Pure Math., vol. 35, part 1, Amer. Math. Soc., Providence, R. I., 1979, pp. 69-83.

9. B. Muckenhoupt and R. Wheeden, Weighted norm inequalities for fractional integrals, Trans. Amer. Math. Soc. 192 (1974), 261-274.

10. E. Sawyer, A characterization of two weight norm inequalities for fractional and Poisson integrais, Trans. Amer. Math. Soc. 308 (1988), 533-545.

11. _— Weighted inequalities for the one-sided Hardy-Littlewood maximal functions, Trans. Amer. Math. Soc. 297 (1986), 53-61.

12. __ Two weight norm inequalities for certain maximal and integral operators, Lecture Notes in Math., vol. 908, Springer, 1982, pp. 102-127. 
13. _ A characterization of a two-weight norm inequality for maximal operators, Studia Math. 75 (1982), 1-11.

14. E. Stein, Interpolation of linear operators, Trans. Amer. Math. Soc. 83 (1956), 482-492.

15. G. V. Welland, Weighted norm inequalities for fractional integrals, Proc. Amer. Math. Soc. 51 (1975), 143-148.

16. W.-S. Young, Weighted norm inequalities for the Hardy-Littlewood maximal function, Proc. Amer. Math. Soc. 85 (1982), 24-26.

Department of Mathematics, University of Alberta, Edmonton, Alberta, CANADA

Department of Mathematics and Statistics, MCMaster University, HamilTON, ONTARIO, CANADA 